\title{
Mulher e negra: dupla vulnerabilidade às DST/HIV/aids
}

To be black and woman: dual vulnerability to STD/HIV/AIDS

Naila Janilde Seabra Santos

Secretaria de Estado da Saúde de São Paulo. Programa Estadual de DST/aids. São Paulo, SP, Brasil.

E-mail: nailaœcrt.saude.sp.gov.br

\section{Correspondência}

Rua Santa Cruz, n 8I, I andar, Gerência de Prevenção-CRT-DST-AIDS.

São Paulo, SP, Brasil. CEP: 04121-000.

\section{Resumo}

O objetivo deste trabalho é discutir os fatores determinantes da vulnerabilidade das mulheres negras a HIV/aids. Pela descrição e análise de dados socioeconômicos, de incidência e mortalidade de aids e da mortalidade de outras patologias, desenha-se o quadro epidemiológico que ressalta as iniquidades em saúde da população negra e, em particular, das mulheres desse segmento populacional. Quando comparadas às mulheres brancas, as negras apresentam, repetidamente, maior risco de adoecimento e morte. A discussão sobre violência sexual e doméstica reitera as disparidades e a maior vulnerabilidade social da mulher negra. As desigualdades socioeconômicas e o racismo institucional são as hipóteses explicativas para a alta vulnerabilidade às DST/aids das mulheres negras. Apenas com uma ampla gama de ações multissetoriais, incisivo enfrentamento do racismo institucional pelo Estado e fortalecimento do movimento social será possível iniciar a longa jornada para se alcançar o propalado princípio de equidade na saúde.

Palavras-chave: Mulher Negra; HIV/aids; Violência; Vulnerabilidade; Racismo Institucional. 


\section{Abstract}

The aim of this paper is to discuss the determinants of the vulnerability of black women to HIV/AIDS. By describing and analyzing socioeconomic data, incidence and mortality by AIDS and other diseases, we have drawn an epidemiological framework that emphasizes health inequities of black people, particularly black women. When compared to white women, black women have repeatedly increased risk of illness and death. The discussion of sexual and domestic violence reiterates disparities and social vulnerability of the black women. Socioeconomic inequalities and institutional racism are explanatory hypotheses for the high vulnerability of black women to STD/AIDS. Only a wide range of multisectoral actions, incisive facing of institutional racism by the governments and strengthening of the social movement will allow the beginning of a long journey to reach the principle of equity in health.

Keywords: Black Women; HIV/AIDS; Violence; Vulnerability; Institutional Racism.

\section{Introdução}

A epidemia de aids é um grande problema de saúde pública no Brasil, e, embora até a atualidade haja mais casos notificados em indivíduos do sexo masculino, a epidemia entre as mulheres apresentou de meados da década de 1980 em diante um crescimento importante. Esse aumento do número de casos na população feminina foi um fenômeno mundial, mas ocorreu de forma mais rápida no Brasil (Bastos, 2009).

Dentre os indicadores que apontam esse crescimento, temos a taxa de incidência de aids no Brasil, que no ano de 1998 era de 25/10o.ooo habitantes homens e de 12,6/100.0oo habitantes mulheres; e em 2010 foi de 22,9/100.00o homens e de 13,2/100.000 mulheres (Brasil, 2011).

No estado de São Paulo, a razão masculino/feminino de casos decresceu de 34:1 em 1985 para 2:1 em 1996, mantendo-se nesse patamar até 2010, subindo para 2,4:1 em 2013 à custa do recrudescimento da epidemia entre os jovens gays. A aids foi a principal causa de mortalidade nas mulheres entre 25 e 34 anos em São Paulo no início da década de 1990 até o fim da década de 2000 e foi a segunda causa de mortalidade nessa faixa etária em 2012. Isso demonstra, inequivocamente, a magnitude com que a aids tem atingido nossa população feminina. Ressalte-se que, na faixa etária de 35 a 44 anos, a aids oscilou entre a primeira e segunda causa de mortalidade dos anos 1990 até 2010, sendo a terceira causa de mortalidade em 2012. Ressalte-se também que, desde 2003, a categoria de exposição heterossexual responde por mais de 80\% dos casos notificados de aids em mulheres (São Paulo, 2014).

Embora a aids seja uma doença de transmissão prioritariamente sexual, provocada por um retrovírus, o vírus da imunodeficiência humana (HIV), a epidemia de aids é dinâmica e multifatorial, e sua ocorrência vai muito além da questão do comportamento sexual, estando relacionada com as condições de vida, gênero, composições etárias e étnicas das populações atingidas.

Desde o início de sua epidemia, houve diversas tentativas de explicação do perfil das pessoas atingidas, iniciando pelo conceito de grupos de risco, seguindo-se os de comportamentos ou situações de 
risco. Entretanto, a dinamicidade da epidemia não se limitou aos grupos e comportamentos de risco descritos, e, com o passar do tempo, outros grupos populacionais foram atingidos. Nesse trajeto, a epidemia de aids abrangeu as mulheres, e ao contrário da fantasia da maioria da população e mesmo dos trabalhadores da saúde, a não obediência aos chamados "grupos de risco" específicos foi facilmente observado na população feminina, na qual uma parcela expressiva das pacientes notificadas apresentava parceria sexual única no momento do diagnóstico e um número de parceiros sexuais na vida que não diferiu muito do relatado pela maioria da população (Santos; Guimarães; Araújo, 2009).

O conceito de vulnerabilidade teve destaque no início da década de 1990 e permitiu uma visão mais ampla sobre como questões relacionadas à desigualdade social, diversidade sexual, raça/etnia, preconceito e discriminação, entre outros fatores que afetam o modo de viver de diversos segmentos populacionais, podem potencializar a disseminação da infecção pelo HIV e a consequente epidemia de aids (Garcia; Souza, 2010).

De acordo com Ayres et al. (apud Garcia; Souza, 2010, p. 10), o conceito de vulnerabilidade é expresso por um "conjunto de aspectos individuais e coletivos relacionados ao grau e modo de exposição a uma dada situação e, de modo indissociável, ao maior ou menor acesso a recursos adequados para se proteger das consequências indesejáveis daquela situação”.

Esse conceito propôs uma abordagem da interrelação dos aspectos individuais, socioeconômicos e de políticas públicas, na busca de hipóteses explicativas da ocorrência da infecção pelo HIV e de sua evolução para o adoecimento e morte por aids (Mann; Tarantola; Netter, 1993; Ayres et al., 2003).

Definiu-se então, dentro do conceito de vulnerabilidade, a subdivisão entre vulnerabilidade individual, social e programática.

Os fatores ligados à vulnerabilidade individual consideram questões como idade, sexo, atividade e práticas sexuais, raça/cor, escolaridade, renda, acesso a serviços de saúde e outros bens sociais. Ou seja, consideram os contextos epidemiológicos e sociodemográficos que determinam um maior ou menor grau de exposição ao HIV/aids dos indivíduos.
À vulnerabilidade social, tangem as questões ligadas ao exercício de cidadania e dos direitos. Aqui se incluem os direitos sexuais e reprodutivos e a diversidade sexual, as questões de gênero e gerações, o pertencimento étnico e racial e seus significados dentro da sociedade.

A vulnerabilidade programática é definida pelo investimento em ações e programas de saúde voltados à prevenção da infecção pelo HIV e à assistência prestada a pessoas vivendo com HIV/ aids, assim como pela possibilidade de acesso dada aos diferentes segmentos da população às ações e equipamentos sociais e de saúde.

As relações entre os indivíduos dentro de uma sociedade e seus equipamentos sociais definem o grau de vulnerabilidade dos indivíduos. Nesse sentido, os estudos de vulnerabilidade buscam compreender a condição das pessoas e/ou dos segmentos populacionais aos quais pertencem. A partir dessa compreensão, o objetivo seria clarear os determinantes sociais dos agravos, trazê-los para a discussão das políticas públicas e construir estratégias de intervenção que pudessem efetivamente alterar esses determinantes (Garcia; Souza, 2010).

No caso da população negra, o meio ambiente que exclui e nega o direito natural de pertencimento coloca o negro brasileiro em condições de vulnerabilidade. Além da inserção social desqualificada/ desvalorizada (vulnerabilidade social) e da invisibilidade de suas necessidades específicas nas ações e programas de assistência e prevenção (vulnerabilidade programática), homens e mulheres negros vivem em um constante estado defensivo. Esse efeito cumulativo de desvantagens individuais, sociais e políticas pode provocar comportamentos inadequados, doenças psíquicas e psicossociais, além das doenças físicas (vulnerabilidade individual). (Pinho et al., 2002, p. 278)

\section{Vulnerabilidade das mulheres - 0 caso especial das mulheres negras}

Desde a década de 1990, diversos estudos apontavam fatores biológicos que explicam a maior 
probabilidade de infecção pelo HIV em mulheres do que em homens, como, por exemplo: o fato de os homens apresentarem maior quantidade de carga viral no esperma do que as mulheres no líquido vaginal; liberação de maior quantidade de secreção sexual masculina ( 5 a $7 \mathrm{ml}$ ) do que feminina (1 a $3 \mathrm{ml}$ ) nas relações sexuais; uso de contraceptivos orais; a alta prevalência de infecções genitais femininas que, mesmo sendo muitas vezes assintomáticas, fragilizam a mucosa vaginal e facilitam a entrada do HIV e outros vírus (Santos, 2002; Vermelho, 1999; Ronald, 1995). Entretanto, ainda que essas informações sejam verdadeiras, não podemos subestimar a forte associação das relações de gênero nas sociedades com a maior vulnerabilidade das mulheres à infecção pelo HIV (Giffin, 1998; Bastos, 200o).

Os riscos de exposição ao HIV se acentuam nas mulheres mais jovens, pela imaturidade do aparelho genital, que leva a uma maior fragilidade da mucosa vaginal.

Bastos (200o) coloca que a determinação socioeconômica e cultural permeia as "regras" de pareamento entre os gêneros na maioria das sociedades e faz mulheres mais jovens habitualmente estabelecerem parcerias sexuais com homens mais velhos, que apresentam maiores prevalências de doenças sexualmente transmissíveis (DST) e de infecção pelo HIV. Ao fazer sexo desprotegido com esses homens, amplia-se o risco de as mulheres jovens adquirirem infecções sexualmente transmissíveis, entre elas o HIV. Além das consequências diretas sobre a saúde das mulheres, essa assimetria de pareamento determina epidemias maiores e mais longas do que seria uma epidemia hipotética, em uma comunidade onde prevalecessem os pareamentos simétricos (Bastos, 200o).

Apesar do avanço dos movimentos feministas nas sociedades ocidentais no que diz respeito aos direitos sexuais e reprodutivos, as mulheres ainda se encontram em grande desvantagem. Mudanças culturais e estruturais são necessárias para que se alcance a tão sonhada igualdade de direitos entre homens e mulheres.

O papel da mulher ainda de submissão com relação às questões sexuais e a responsabilização das mulheres no que diz respeito às questões re- produtivas dificultam o diálogo com seus parceiros e aumentam sua vulnerabilidade.

A discussão sobre a epidemia de aids e seu enfrentamento passam pela compreensão das normas de gênero nas sociedades e como elas influenciam e determinam as relações entre homens e mulheres. A proposição/negociação do uso do preservativo esbarra em questões delicadas, sobre as quais é difícil dialogar, ainda mais quando se estabelece uma relação de afeto e/ou de poder entre os indivíduos. Assim, a maior vulnerabilidade das mulheres à infecção pelo HIV passa por questões complexas, que vão desde o comportamento sexual "esperado" para cada gênero, os papéis sociais a ser cumpridos por homens e mulheres e a dinâmica de poder entre os gêneros (Barbosa, 1999).

Embora o diálogo sobre uso de preservativos e prevenção das DST seja difícil mesmo para as mulheres jovens e solteiras, para as mulheres casadas é ainda mais complicado, uma vez que a sugestão de atividades de prevenção ou uso de preservativos para elas pressupõe uma quebra de confiança com seu companheiro e pode trazer dúvidas com relação a sua própria fidelidade (Garcia; Souza, 2010).

Analisando os dados da pesquisa realizada em 1998 sobre o comportamento sexual da população brasileira e percepções do HIV/aids segundo raça/ cor e sexo, Pinho et al. (2002) colocam que a maior proporção de usuários de preservativos foi encontrada entre brancos e negros não unidos, e a menor adesão, entre aqueles com parcerias estáveis e eventuais, especialmente entre jovens negras.

Entre homens e mulheres brancas e solteiras, não houve diferença quanto à proporção de pessoas que referiram uso de camisinha (57\%). Para a população negra, $66 \%$ dos homens solteiros referiam uso de preservativos, contra apenas $45 \%$ das mulheres negras solteiras.

A comparação dentro de cada sexo mostrou que não houve diferença entre o não uso de preservativos entre homens jovens brancos e negros. Já entre as mulheres jovens, o não uso apareceu em uma proporção maior para as negras (72\%) do que para as brancas (58\%).

Quanto à atividade sexual, $72 \%$ dos homens brancos e $67 \%$ dos homens negros se declararam 
sexualmente ativos. A vida sexual ativa foi relatada por uma proporção maior de mulheres brancas (59\%) do que de mulheres negras (49\%).

Na faixa etária de 16 a 24 anos, o uso de preservativos foi citado por $33 \%$ dos homens brancos, $26 \%$ dos homens negros, $25 \%$ das mulheres brancas e $16 \%$ das mulheres negras. Ainda que a proporção de uso seja baixa para todos os extratos apresentados, a menor proporção de uso é das mulheres negras.

Em estudo sobre os determinantes de vulnerabilidade de mulheres negras ao HIV, Albuquerque, Moço e Batista (2011) colocam que, de 1.221 mulheres negras entrevistadas, apenas $23 \%$ referiam uso consistente de preservativos, e 54,5\% referiram nunca utilizá-los.

Estudando a vulnerabilidade de meninas adolescentes em uma comunidade pobre no Rio de Janeiro, Taquette (2011) cita uma proporção muito maior de histórico de DST entre as afrodescendentes.

Não há dúvida de que as infecções sexualmente transmissíveis são mais comuns nos segmentos mais pobres da população. 0 menor acesso a serviços e a tratamentos eficazes da população desfavorecida economicamente favorece a cronificação dessas infecções, que potencializam o risco de aquisição do HIV (Bastos, 2000).

Santos et al. (2009), em estudo sobre os contextos de vulnerabilidade das mulheres brasileiras ao HIV, apontaram o uso de drogas, o início da vida sexual mais precoce, a baixa aderência ao uso de preservativos, a maior proporção de histórico de DST e de violência sexual como os fatores que apresentaram diferenças significantes, do ponto de vista estatístico, entre as mulheres vivendo com HIV e as demais mulheres pesquisadas.

A violência nas relações amorosas ou íntimas afeta especialmente as mulheres e está associada com as relações de gênero e sua hierarquia "naturalmente" estruturada em bases desiguais, que reservam às mulheres um lugar de submissão e de menor valia nas sociedades.

Nesse contexto, explica-se a ocorrência predominante da violência contra a mulher no espaço doméstico, tanto a violência sexual quanto a física e a psicológica. Os agressores, em sua grande maioria, são conhecidos, sendo identificados com maior frequência os pais biológicos, padrastos, maridos, namorados ou ex-companheiros; irmãos, parentes próximos e vizinhos (Blake et al., 2014).

No tocante às mulheres negras, essas relações são ainda mais complexas, se pensarmos que na escala hierárquica da sociedade é reservado a elas o espaço de menor poder, pois se somam à dinâmica já perversa das relações de gênero, outras formas de dominação e desigualdade sociais advindas das relações étnico/ raciais e de uma situação socioeconômica, na maioria das vezes menos privilegiada.

Sueli Carneiro (2003, p.14), falando sobre a mulher negra, coloca:

há uma forma específica de violência que constrange o direito à imagem ou a uma representação positiva, limita as possibilidades de encontro no mercado afetivo, inibe ou compromete o pleno exercício da sexualidade pelo peso dos estigmas seculares, cerceia o acesso ao trabalho, arrefece as aspirações e rebaixa a autoestima [...] a exclusão simbólica, a não-representação ou distorções da imagem da mulher negra nos meios de comunicação são formas de violência tão dolorosas, cruéis e prejudiciais que poderiam ser tratadas no âmbito dos direitos humanos.

Em estudo americano sobre o julgamento de agressores sexuais, Crenshaw (2002) sugere que a identidade racial da vítima assume um papel significativo na determinação dos resultados e que há evidências de que os jurados podem ser levados, pela propaganda sexualizada, a acreditar na maior probabilidade de que mulheres racializadas consintam em ter relações sexuais em circunstâncias que eles achariam pouco prováveis se a vítima não fosse de uma minoria racial.

Estudos que investigaram as diversas formas de violência contra as mulheres demonstram que não apenas a violência sexual, mas também a violência física, aumenta a vulnerabilidade das mulheres às DST/aids.

A violência doméstica e relações em que o parceiro tem controle excessivo sobre as companheiras estão associadas, segundo estudos com mulheres africanas, a uma prevalência da infecção por HIV $50 \%$ mais alta (WHO, 2008). 
Outro aspecto a ser considerado é que quando a mulher solicita um exame de HIV, compartilha seu status sorológico com o parceiro, solicita o uso do preservativo na relação sexual com o parceiro fixo para prevenir doenças, ou quando precisa tomar decisões sobre uma gravidez até o momento em que busca tratamento para si ou para o parceiro ou para a prevenir a transmissão vertical do HIV para seu bebê, ela também pode ser alvo de violência por sua condição sorológica (Campbell et al., 2008).

Santos et al. (2009) verificaram que, embora apenas $2 \%$ das mulheres vivendo com HIV tenham relatado a violência sexual como categoria de exposição ao vírus, $22,1 \%$ dessas mulheres apresentam histórico de violência sexual alguma vez na vida, enquanto em mulheres soronegativas esse índice cai para $13,2 \%$.

Essa diferença mostrou significância estatística, evidenciando o fato das mulheres que vivem com HIV sofrem mais violência sexual na vida, o que de alguma forma as torna mais vulneráveis às DST/aids.

Estudos na África do Sul, na Uganda e na Índia, embora não consigam definir os complexos caminhos entre violência doméstica e HIV, concluíram que a violência é causa e consequência da infecção pelo HIV, e estudos prospectivos mostram associação entre companheiro violento e/ou violência sexual e incidência de HIV/aids (Dunkle et al., 2004; GarciaMoreno et al., 2006; Silverman et al., 2007).

A maior prevalência da infecção pelo HIV entre mulheres vítimas de violência doméstica varia globalmente, mas sua associação aparece em todos os países, de forma independente e simultânea.

Estudos entre várias populações apoiam a existência de uma relação temporal e biologicamente complexa entre o risco de HIV e exposição à violência na vida, o que pode representar um fator de complicação coadjuvante nas já complexas relações de gênero e decisões sexuais.

O uso abusivo de álcool por parte dos companheiros e situações de violência doméstica estão relacionados entre si e com o fato de as mulheres serem mais frequentemente submetidas a relações sexuais forçadas (Phorano; Nthomang; Ntseane, 2012). Entretanto, futuros estudos prospectivos são fundamentais para investigar as questões de causalidade e temporalidade que existem entre violência doméstica e infecção pelo HIV.

Sendo as violências sexuais e domésticas fatores determinantes da vulnerabilidade das mulheres, as mulheres negras aparecem novamente, segundo diversos estudos, em posição de desvantagem:

Os primeiros estudos sobre violência contra a mulher mostravam que o evento acontecia em todas as classes sociais. Porém, há evidências de sobreposição entre os sistemas de dominação e exploração constituídos pelas relações de gênero, etnia e classe social, acarretando às mulheres pobres e negras uma carga mais pesada e maior exposição às violências (Kronbauer; Meneghel, 2005, p. 700).

No mesmo estudo, os autores colocam que as mulheres negras referiram maior prevalência de violência psicológica (59\%) e física (40\%) do que as brancas ( $51 \%$ e $36 \%$, respectivamente).

Um estudo qualitativo com mulheres de 16 a 55 anos de idade de comunidades remanescentes de quilombos em Alagoas apontou que um acentuado número de mulheres é submetido à violência doméstica em diversos níveis, incluindo físico e sexual. As mulheres quilombolas relataram ser vítimas de preconceito racial, sofrendo humilhação na rua, na escola, em festas e inclusive no atendimento em postos de saúde (Riscado; Oliveira; Brito, 2010).

Os autores concluem que o alto grau de vulnerabilidade à infecção por DST/aids e outros agravos ocorrem pela ausência de uma política de saúde mais efetiva e de um trabalho educativo nessas comunidades.

Schraiber, D’Oliveira e França Junior (2008) relatam, em estudo sobre a violência entre parceiros sexuais íntimos, uma maior frequência de episódios de violência sexual entre os negros de ambos os sexos e que as mulheres negras referiram ter mais relações sexuais por medo do parceiro do que as mulheres brancas.

Maiores proporções de violência sexual apareceram nesse estudo para pessoas com baixos níveis de escolaridade e de renda, e a ocupação que sofreu maior número de agressões foi a de empregada doméstica. Condições estas ligadas à grande parcela das mulheres negras. 
Além desses fatores, ser separada ou viúva aumentava a probabilidade de ser vítima de violência sexual. Ressalte-se que as mulheres negras apresentam, em mais de um estudo, número menor de parceiros/cônjuges do que as mulheres brancas (Schraiber et al., 2002; Pinho et al., 2002).

Em estudo observacional de corte transversal com adolescentes femininas menores de 15 anos e sexualmente ativas, atendidas em um ambulatório público de ginecologia no Rio de Janeiro, os fatores associados à atividade sexual desprotegida foram: início da atividade sexual antes dos 13 anos, exploração sexual comercial, múltiplas parcerias sexuais, violência intrafamiliar, atraso escolar, ser da raça/cor negra, gravidez não esperada e DST (Taquette; Teixeira, 2010).

Esse estudo destaca ainda que a sinergia entre pobreza, baixa escolaridade e baixa autoestima diminui as chances de elaboração, por parte das adolescentes, de mecanismos de autodefesa, expondoas à possibilidade de revitimização.

A violência, que é resultante das desigualdades de gênero e raça/etnia, está relacionada à feminização da aids, pela dificuldade que confere às mulheres, em especial as jovens e negras, de negociar com seus parceiros práticas sexuais seguras, mas também pelas violências sexual e institucional (Taquette, 2011).

As mulheres negras sofrem dupla discriminação, pois vivem em condições desiguais de gênero (são estigmatizadas por serem mulheres) em uma sociedade que perpetua as desigualdades étnico/raciais (estigmatizadas por serem negras) (Diniz, 2007).

Os atos de discriminação intencional não se limitam à violência sexual. No mercado de trabalho, na educação e em vários âmbitos sociais, as mulheres negras são sujeitas a discriminações e opressões por não pertencerem nem ao gênero nem ao grupo étnico e racial dominante na sociedade. Isso sem dúvida configura uma dupla exclusão. De fato, as mulheres negras entram em desvantagem quando competem por um emprego com as mulheres brancas e/ou com indivíduos do sexo masculino (Crenshaw, 2002).

Na nossa sociedade, os jovens negros são particularmente afetados pelas desigualdades socioculturais. São eles que apresentam os mais baixos níveis de escolaridade, as maiores dificuldades para entrar no mercado de trabalho, especialmente em funções mais valorizadas, e os que sofrem maior discriminação nos serviços de saúde. Dentro dessa categoria, destacam-se ainda mais as dificuldades das jovens negras, que, entre homens e mulheres brancos e negros, são as que apresentam os menores índices de escolaridade a as mais baixas rendas mensais (Pinho et al., 2002; Cunha, 2010).

Assim, os programas de saúde e educação voltados ao enfrentamento das DST/aids deveriam abandonar as estratégias de caráter eminentemente informativo e focar as possibilidades concretas de intervenção, levando em conta a vulnerabilidade conferida pelas desigualdades sociais, pelo pertencimento étnico-racial e de gênero (Pinho et al., 2002).

\section{Um olhar sobre os dados epidemiológicos}

A introdução do quesito raça/cor nas estatísticas de saúde no Brasil foi justificada como forma de desnudar o mito da democracia racial (Adorno; Alvarenga; Vasconcellos, 2004), permeado pela falta de informações específicas sobre cor ou raça/etnia nos dados oficiais do setor saúde, o que dificulta o monitoramento das condições de saúde da população negra e do racismo institucional.

Em 1996, o quesito cor foi introduzido no SIM e no SINASC, respectivamente os sistemas de informação sobre mortalidade e sobre nascidos vivos utilizados no Brasil. Em 200o, foi incluído no SINAN, sistema de informação dos agravos de notificação.

As diferenças na mortalidade da população negra com relação à população branca têm sido descritas em diversos estudos brasileiros. Entre tantas diferenças, destacam-se as causas externas (homicídios, acidentes de trânsito, quedas, afogamentos, suicídio etc.), as doenças cerebrovasculares e a mortalidade materna. As duas últimas causas figuram entre as causas de óbito evitáveis e inegavelmente relacionadas com a falta de acesso e a baixa qualidade da atenção oferecida nos equipamentos de saúde, fragilizados por vários problemas estruturais como um todo e particularmente inadequados na atenção à saúde da população negra (Batista, 2002; Adorno, 2004; Santos; Guimarães; Araújo, 2007). 
Ao mensurar a desigualdade racial na mortalidade por causas externas em mulheres residentes em Recife, Santos, Guimarães e Araújo (2007) apontam a necessidade de outras pesquisas que permitam aprofundar a interseção entre violência contra a mulher e recorte racial. Quanto às doenças infecciosas e parasitárias, o risco foi mais elevado em mulheres adultas negras, destacando-se, entre as causas específicas desse capítulo, a aids e a tuberculose.

Tanto no Brasil quanto em outras partes do mundo, ser negro é estar submetido a condições desfavoráveis de viver e morrer sem que isso seja levado em consideração, e, para estes indivíduos, o fato de ser do sexo feminino é ainda mais complicado. Exemplificando, enquanto 32\% dos homens HIV-positivos da Casa de Detenção do Estado de São Paulo em 1996 eram negros (Kallás, 1996), entre as soropositivas da Penitenciária Feminina, 77\% eram mulheres negras. Assim, além de as prisões concentrarem indivíduos negros e pobres que não puderam atingir os patamares mínimos para o acesso a bens culturais e/ou de serviços, concede-lhes o direito à participação no grupo dos "especialmente vulneráveis” às doenças infecto-contagiosas, como é o caso do HIV/AIDS. (Lopes et al., 2001, p. 1477)

Em artigo sobre a magnitude e as tendências da epidemia norte-americana de aids, Rosenberg (1995 apud Bastos; Szwarcwald, 200o) destaca a questão da vulnerabilidade associada à raça/etnia. Outros autores ponderaram que o recorte dos dados pela renda diluía o peso da origem étnica como variável explicativa, uma vez que não há qualquer fator biológico que explique a maior vulnerabilidade de negros e latinos à infecção pelo HIV. Rosenberg, mesmo reconhecendo a relevância das críticas, observou que, mesmo fazendo o pareamento dos indicadores socioeconômicos no que tange à hepatite B e o HIV/aids, sempre permanece um "resíduo" de maiores taxas de incidência nas populações negras e latinas, de magnitude variável.

Em 2002, ao apresentar sua tese de Doutorado, Batista chama atenção para os diferenciais dos coeficientes de mortalidade por aids no estado de São
Paulo em 1996, entre homens e mulheres brancos e pretos. Enquanto os homens pretos apresentavam um coeficiente de mortalidade de 25,92 por 100.000 habitantes, para os homens brancos esse indicador foi 14,44/100 mil. Já as mulheres pretas apresentavam um coeficiente de mortalidade de 11,39/100 mil, mais do que o dobro do mesmo indicador para as mulheres brancas, que foi de 4,92/10o mil.

Interessante observar nos dados do Sistema de Informação Ambulatorial e Hospitalar do SUS, no ano de 2012, que as proporções de internação por doenças infecciosas, entre as quais se inclui a aids, são muito maiores para a população negra (39,3\% para homens e $40,3 \%$ para mulheres) do que para a população branca (23,5\% para homens e $23 \%$ para mulheres). A proporção de óbitos decorrentes de internações por gravidez, parto ou puerpério foi de $0,15 \%$ nas mulheres brancas e mais que o dobro (o,37\%) nas mulheres negras (Brasil, SAi/SUS, 2012).

Volochko (2010), em estudo sobre os determinantes da mortalidade materna, coloca que no Brasil, em 2003, a mortalidade das mulheres pretas foi maior do que das brancas em todas as faixas etárias, chegando a ser três vezes maior na faixa de 30 a 39 anos de idade, e embora em uma proporção um pouco menor, a mortalidade das mulheres pardas também é maior do que das brancas, em todas as faixas etárias, exceto na de 5-14 anos de idade.

0 diferencial de mortalidade materna (mortes relacionadas à gestação, parto ou puerpério) entre mulheres brancas e negras impacta sempre pela sua dimensão. Destaca-se o fato de que, no estado de São Paulo, a taxa de mortalidade materna entre mulheres pretas foi 10,34 vezes maior do que entre mulheres brancas (Volochko, 2011). Em Recife, a mortalidade materna nas mulheres brancas foi de 37,73; e quase seis vezes maior nas mulheres pretas, 212,80. Essa diferença foi colocada como "escandalosa e criminosa” (Santos; Guimarães; Araújo, 2007).

A Vigilância Epidemiológica de aids do estado de São Paulo, na publicação com a fundação SEADE Dados para repensar a aids no estado de São Paulo, de 2010, coloca novamente em evidência os diferenciais relativos ao quesito raça/cor, como se vê na tabela extraída da página 52 do trabalho de Guibu, Tayra e Carmo (2010) (Tabela 1): 
Tabela I - Número médio de casos e taxas médias de incidência de aids, por sexo, segundo raça/cor, estado de São Paulo (2003-2005)

\begin{tabular}{|c|c|c|c|c|}
\hline \multirow{2}{*}{$\begin{array}{c}\text { Raça/ } \\
\text { cor }\end{array}$} & & \multicolumn{2}{|c|}{ Sexo } & \multirow{2}{*}{ Total } \\
\hline & & masculino & feminino & \\
\hline \multirow[t]{2}{*}{ Branca } & $\mathrm{n}$ médio casos & 2.943 & 1.596 & 4.539 \\
\hline & $\begin{array}{l}\text { Taxa média de } \\
\text { Incidência }\end{array}$ & 22,00 & 11,1 & 16,4 \\
\hline \multirow{2}{*}{$\begin{array}{l}\text { Preto- } \\
\text { pardo }\end{array}$} & $n$ médio casos & 1.297 & 853 & 2.150 \\
\hline & $\begin{array}{l}\text { Taxa média de } \\
\text { Incidência }^{(1)}\end{array}$ & 23,0 & 15,5 & 18,7 \\
\hline \multirow[t]{2}{*}{ Amarela } & $\mathrm{n}$ médio casos & 29 & 14 & 43 \\
\hline & $\begin{array}{l}\text { Taxa média de } \\
\text { Incidência }{ }^{(1)}\end{array}$ & 14,8 & 6,4 & 10,3 \\
\hline \multirow[t]{2}{*}{ Total } & média anual ESP & 4.685 & 2.731 & 7.417 \\
\hline & $\begin{array}{l}\text { Taxa média de } \\
\text { Incidência (1) }\end{array}$ & 24,3 & 13,6 & 18,9 \\
\hline
\end{tabular}

$\mathrm{Na}$ análise dos dados, as autoras calcularam o risco relativo (RR) - uma razão entre coeficientes que mede o risco de ocorrência de um dado evento em uma população em comparação a outra. Assim, observaram que o risco de ter aids dos homens negros (pretos + pardos) foi $5 \%$ maior do que o dos homens brancos $(\mathrm{RR}=1,05)$. As mulheres negras (pretas + pardas) apresentaram $\mathrm{RR}=1,4$ quando comparadas às mulheres brancas, o que representa um risco de incidência de aids 40\% maior nas mulheres negras.

Considerando os dados de 2004 a 2006, a Vigilância Epidemiológica do CRT elaborou a tabela a seguir (Tabela 2):

Fonte: Base Integrada Paulista de aids (BIP-AIDS)

\section{Tabela 2 - Casos e coeficientes de incidência (por 100.000) de aids, segundo raça/cor e sexo, no $\operatorname{ESP}(2004$ a 2006*)}

\begin{tabular}{|c|c|c|c|c|c|c|c|c|c|c|c|c|}
\hline \multirow{3}{*}{$\begin{array}{l}\text { Ano de } \\
\text { Diagnóstico }\end{array}$} & \multicolumn{12}{|c|}{ Raça/Cor } \\
\hline & \multicolumn{2}{|c|}{ Branca } & \multicolumn{2}{|c|}{ Pre } & \multicolumn{2}{|c|}{ Pard } & \multicolumn{2}{|c|}{ Preta+Pard } & \multicolumn{2}{|c|}{ Amarela } & \multicolumn{2}{|c|}{ Indígena } \\
\hline & $n$ & coef & $n$ & coef & $n$ & coef & $n$ & coef & $n$ & coef & $n$ & coef \\
\hline \multicolumn{13}{|l|}{ masculino } \\
\hline 2004 & 2850 & 21,4 & 462 & 48,2 & 849 & 18,3 & 1311 & 23,4 & 26 & 13,6 & I & 3,5 \\
\hline 2005 & 2680 & 19,8 & 374 & 38,5 & 850 & 18,1 & 1224 & 21,6 & 31 & 16,0 & 3 & 10,5 \\
\hline 2006 & 2560 & 18,7 & 378 & 38,5 & 803 & 16,9 & 1181 & 20,6 & 20 & 10,2 & 5 & 17,3 \\
\hline \multicolumn{13}{|l|}{ feminino } \\
\hline 2004 & 1533 & 10,7 & 313 & 31,3 & 555 & 12,5 & 868 & 15,9 & 14 & 6,4 & - & - \\
\hline 2005 & 1399 & 9,7 & 318 & 31,4 & 585 & 12,9 & 903 & 16,3 & 14 & 6,3 & 3 & 9,9 \\
\hline 2006 & 1296 & 8,8 & 233 & 22,7 & 512 & $\mathrm{II}, 2$ & 745 & 13,3 & 9 & 4,0 & 3 & 9,8 \\
\hline \multicolumn{13}{|l|}{ total } \\
\hline 2004 & 4383 & 15,9 & 775 & 39,6 & 1404 & 15,4 & 2179 & 19,7 & 40 & 9,7 & I & I,7 \\
\hline 2005 & 4079 & 14,6 & 962 & 34,9 & 1435 & 15,6 & 2127 & 19,0 & 45 & 10,8 & 6 & 10,2 \\
\hline 2006 & 3856 & 13,6 & 611 & 30,4 & 1315 & 14,1 & 1926 & 17,0 & 29 & 6,9 & 8 & 13,4 \\
\hline
\end{tabular}

Fonte: SINAI - VE - Prog

IBGE: Pesquisa Nacional por Amostras de Domicílios - PNDA, Fundação SEADE

* sem os casos ignorados para a raça/cor

Analisando os dados apresentados nessa tabela, vê-se que em ambos os sexos as taxas de incidência de negros (pretos e pardos) foram maiores do que em brancos.

Essas diferenças se acentuam se tomarmos os indivíduos de cor preta separadamente. Nos anos de 2004, 2005 e 2006, enquanto os coeficientes de incidência de aids entre homens pretos foram aproximadamente o dobro dos coeficientes entre homens brancos, os coeficientes das mulheres negras foram praticamente o triplo dos apresentados pelas mulheres brancas.

Em 2006, o RR da população negra, em comparação à branca, foi 1,25, ou seja, os negros tiveram 25\% mais chances de ter aids do que brancos.

Fazendo a análise por sexo, entre os homens, essa diferença foi menor, pois o RR resultou em 1,1 no ano de 2006. 
Ao calcular o RR entre mulheres pretas e pardas com relação às mulheres brancas, nota-se que as pretas e pardas possuíram um risco de ter aids 51\% maior do que as brancas.

O Programa Municipal de São Paulo, utilizando os recortes raciais da população do Censo Demográfico do IBGE, de 2010, calculou a incidência de aids no município de São Paulo para o mesmo ano por raça/ cor e encontrou coeficientes por 100.ooo habitantes de 39,7 para os pretos, 21,2 para os pardos e 18 para os brancos residentes na cidade (São Paulo, 2012).

Ainda para 2010, o programa municipal calculou os coeficientes de mortalidade por aids para pretos (13,2/100.00o), pardos (7,7/100.00o) e brancos (6,9/10o.ooo) no município de São Paulo.

Detalhando a proporção de óbitos por raça/cor, sexo e escolaridade em 2010 no município de São Paulo, a despeito do alto número de dados ignorados, observaram que para os homens, entre os analfabetos e na faixa de 4 a 7 anos de escolaridade, os pretos e pardos apresentaram maiores proporções de óbitos; na faixa de 12 anos e mais de estudo, a maior proporção de casos foi em brancos. Nas demais faixas de escolaridade, 1 a 3 anos e 8 a 11 anos de estudo, as proporções foram semelhantes.

As mulheres com aids apresentaram globalmente uma escolaridade bem menor do que os homens. As mulheres pretas apresentam maior proporção de óbitos entre as analfabetas e nas faixas de 1 a 4 anos; de 4 a 7 e de 8 a 11 anos de escolaridade, sendo maior a proporção de brancas apenas na faixa de escolaridade de 12 anos e mais. As mulheres pardas apresentaram maior proporção de casos em todas as faixas de 1 a 11 anos de estudo, se comparadas com as mulheres brancas (São Paulo, 2012).

O estudo de Santos, Guimarães e Araújo (2007), que discutiu o perfil da mortalidade na cidade de Recife, descreve que, no tocante à mortalidade por todas as causas em mulheres, a proporção de óbito das mulheres negras foi maior que das mulheres brancas em todas as faixas etárias, exceto na faixa de 50 a 59 anos, observando uma maior concentração de óbito nas negras em idade precoce, sendo que, na faixa etária de 20 a 29 anos, as causas maternas, externas e por aids ocuparam posição de destaque.
Com exceção do câncer de mama, todas as outras causas de mortalidade específica estudadas apresentaram coeficiente de mortalidade maior em mulheres negras do que em brancas, com razão de taxas variando entre 1,3\% (aids) e 9,7\% (homicídios). 0 risco de morte por doença hipertensiva foi duas vezes maior em negras do que em brancas. 0 câncer de colo de útero, sabidamente associado a uma infecção sexualmente transmissível (HPV), ficou entre as cinco causas específicas na faixa etária entre 40 e 49 anos, com maior risco de morte de mulheres negras.

As desigualdades raciais foram ressaltadas em todas as formas de mortalidade por causas externas, sendo o risco de morte por acidente das mulheres negras duas vezes maior em comparação com as brancas. 0 risco de morte por suicídio das mulheres negras, na faixa etária de 20 a 29 anos, foi quatro vezes superior ao das mulheres brancas. Saliente-se que os homicídios estão na primeira posição como causa específica de óbito de jovens negras. Na faixa etária de 20 a 29 anos de idade, o risco das negras foi 40 vezes maior do que das brancas, e na de 30 a 39 anos, foi 9 vezes superior ao das brancas (Santos; Guimarães; Araújo, 2007).

As autoras destacam que, apesar do claro predomínio da mortalidade por causa externa entre os homens, particularmente os jovens negros, desde 1980 vem crescendo a participação dos homicídios na mortalidade das mulheres brasileiras.

Todos esses dados nos devolvem, com força, a reflexão sobre violência, DST/aids e vulnerabilidade das mulheres, especialmente das mulheres negras e jovens. É importante reconhecer as questões de gênero e raça/cor e lidar com o diferencial de poder entre homens e mulheres/brancos e negros como fator determinante das doenças e da transmissão do HIV.

\section{"Escalas" de risco na saúde e na aids}

Se teorizarmos sobre os dados epidemiológicos descritos, considerando o recorte de sexo e os dois grupos étnico-raciais colocados - população branca e população negra -, seria possível construir "escalas de risco" para alguns eventos em saúde, nos quais o menor risco de adoecimento e morte seria a base, e o maior seria o ápice da "escala”. 
No tocante à mortalidade por causas externas, as bases das escalas femininas e masculinas seriam da população branca, e o ápice, reservado às mulheres e homens negros.

Para a mortalidade materna, a base seria ocupada pelas mulheres brancas, e o ápice, muitas vezes maior do que a base, seria ocupada pelas mulheres negras.

Com relação à infecção pelo HIV e a aids, as escalas de incidência e mortalidade seriam iguais, sendo novamente as bases ocupadas pela população branca e os ápices pela população negra, com um diferencial importante da escala feminina com relação à masculina, dado que os coeficientes de mortalidade e o risco relativo de adoecimento por aids é muito maior entre as mulheres pretas com relação às brancas do que entre os homens pretos e brancos.

Em todas essas escalas e em muitas outras, salta aos olhos a constante posição de desvantagem das mulheres negras no âmbito da saúde (Kalckmann, 2007). Os indicadores de aids apenas reiteram essa desvantagem.

O HIV/AIDS expressa a realidade da vulnerabilidade social, o trabalho da biopolítica sobre os corpos, particularmente no duplo vínculo de raça e gênero. A prevenção precisa empoderar aos sujeitos para se efetivar. A adesão ao tratamento tem que vencer os estigmas vinculados a gênero, sexualidade, raça e HIV (Lopez, 2011, p. 601).

\section{Desigualdades, disparidades em saúde e racismo institucional}

As relações desiguais se reproduzem de maneira contundente quando consideramos a questão étnico-racial que, aliada às questões de gênero, tem um efeito devastador na saúde das mulheres negras.

Pensando nas disparidades raciais dos indicadores de mortalidade de aids apresentados por Batista (2002), especialmente no que concerne às mulheres, alguns questionamentos emergem. A primeira hipótese explicativa que surge, para todos os dados que evidenciam as desvantagens da população negra, é que a questão está ligada à pobreza, a qual está submetida à maior parcela dessa população, e não a questões étnico-raciais.

Sabendo-se que a aids não apresenta diferenciais de evolução e prognóstico entre as diversas raças, indaga-se, então, como as diferenças socioeconômicas explicariam indicadores de mortalidade por aids tão díspares entre a população branca e a população negra, sendo esta uma doença na qual mais de $90 \%$ dos pacientes são atendidos nos serviços públicos do Sistema Único de Saúde (SUS), que têm por princípios a universalidade e a equidade dos atendimentos.

Há que se lembrar, ainda, da proposta de humanização das práticas médicas no SUS. Entretanto, parece inegável, a partir dos dados epidemiológicos, não só de mortalidade, mas também de acesso a serviços e qualidade do atendimento, que algo ainda não mensurado e talvez "não mensurável" ocorre dentro das instituições de saúde, levando a resultados tão díspares dos indicadores de saúde segundo a raça/cor dos cidadãos brasileiros.

O uso das desigualdades econômicas como hipótese explicativa das iniquidades raciais joga uma "cortina de fumaça" sobre a exclusão e a intolerância a que a população negra é submetida nos mais diversos setores da sociedade, incluindo os equipamentos de saúde (Taquette, 2011).

Esse argumento é desmontado por diversos estudos (Santos; Guimarães; Araújo, 2007; Lopes, 2003; Batista, 2002) que demonstram o forte componente de discriminação racial embutido nas desigualdades sociais, desarticulando a hipótese de que os fatores ligados ao racismo podem sempre ser explicados pelas condições socioeconômicas desprivilegiadas de grande parcela da população negra.

Nesse ponto, não é possível nos furtarmos de revisitar o conceito de racismo institucional.

Racismo institucional é o fracasso coletivo de uma organização em prover um serviço apropriado e profissional às pessoas em razão de sua cor, cultura, ou origem étnica. Ele se manifesta em normas, práticas e comportamentos discriminatórios adotados no cotidiano de trabalho, os quais são resultantes da ignorância, da falta de atenção, do 
preconceito ou de estereótipos racistas. Em qualquer caso, o racismo institucional sempre coloca pessoas de grupos raciais ou étnicos discriminados em situação de desvantagem no acesso a benefícios gerados pelo Estado e por demais instituições e organizações (Dias; Giovanetti; Santos, 2009, p. 16).

Conclui-se que as desigualdades socioeconômicas e culturais e o racismo institucional são hipóteses plausíveis para explicar a alta vulnerabilidade às DST/aids das mulheres negras.

\section{A urgência em definir as mulheres negras como população prioritária nas políticas públicas de DST/aids}

A possibilidade de infecção pelo HIV está presente em qualquer relação sexual na qual um dos parceiros esteja infectado, independente do sexo, identidade sexual, gênero, raça ou questão geracional, e todas as parcelas da população merecem algum cuidado/intervenção na linha da prevenção.

A prevalência da infecção pelo HIV na população adulta no Brasil varia em torno de o,5\% há vários anos (Szwarcwald et al., 20o8), segundo os estudossentinela efetuados pelo Ministério da Saúde.

Em algumas populações específicas, os dados sobre a prevalência da infecção pelo HIV são bastante diferentes. Estudos mostram uma prevalência da infecção pelo HIV de 10,5\% em homens que fazem sexo com homens (HSH) (Kerr, 2009); 5,9\% em pessoas usuárias de drogas injetáveis (PUDI) (Bastos, 2009) e 4,9\% em profissionais do sexo (Szwarcwald, 2009).

Essas altas prevalências embasam o discurso de que temos no Brasil uma epidemia concentrada e apontam por que, inequivocamente, essas populações têm sido consideradas como prioritárias nas políticas públicas voltadas ao HIV/aids no país.

Sem dúvida, a frequência de um evento é um dos principais critérios para estabelecer prioridades em saúde pública. Entretanto, não é o único.

Os crescentes indicadores de incidência e os altos coeficientes de mortalidade por aids colocam a população negra em alto grau de vulnerabilidade, e nesse segmento populacional se destaca enfatica- mente a magnitude com que a aids tem atingido as mulheres negras.

A análise dos dados epidemiológicos e a produção de informações que repetidamente reiteram a vulnerabilidade das mulheres negras ao HIV/aids não podem ser desconsideradas.

Algumas medidas impostas pela lógica dos dados têm sido propostas pelo poder público (Lopez, 2011). Entre elas, a campanha da aids de 2005, denominada "aids e Racismo, o Brasil tem que viver sem preconceito", a "Política Nacional de Saúde Integral da População Negra”, a formação dos comitês técnicos estaduais e regionais de saúde da população negra, a criação de metas especificamente voltadas às mulheres negras para enfrentar a epidemia entre as mulheres, entre outras.

Entretanto, se essas medidas representaram um avanço teórico na proposição de políticas públicas, do ponto de vista prático, não foram ainda capazes de impactar os indicadores de saúde da população negra.

E embora os estudos venham apontando, desde o início dos anos 200o, o perfil das desigualdades raciais no acesso a serviços e a procedimentos em saúde e os diferenciais de mortalidade e morbidade em várias situações, entre elas a aids, esses dados ainda não foram suficientes para definir a população negra, particularmente o segmento das mulheres negras, como uma população prioritária para as intervenções dos programas de DST/aids, arraigados na definição das populações classicamente tidas como vulneráveis, como HSH, PUDI e profissionais do sexo.

É preciso incluir, incisivamente, as mulheres negras na agenda dos programas de DST/aids.

\section{Considerações finais}

A violência racial e de gênero representam obstáculos para a equidade em saúde e uma violação dos direitos humanos, sendo fundamental articular diversos setores para seu enfrentamento.

Entretanto, é fundamental que as mulheres se conscientizem que são vítimas de diversos tipos de violência, fato que muitas vezes é negado e naturalizado pelos ideais machistas das sociedades (Meneghel, 2005; Oliveira; Meneghel; Bernardes, 
2009). A continuidade dessa luta e a resolução das questões de violência contra a mulher devem ser efetuadas pelo Estado, pois representa uma violação dos seus direitos humanos. O Estado tem como responsabilidade a adoção de medidas para conter ou eliminar as violências praticadas na sociedade, particularmente contra as mulheres discriminadas por sua origem étnico-racial, geração ou qualquer outro aspecto que, injustamente, designa determinados segmentos sociais como inferiores.

Pelo caráter multifatorial de sua determinação, a implementação de uma política voltada para a redução da violência sexual e doméstica deve contemplar ações interssetoriais. Deve-se salientar que a promoção da saúde, assim como a redução dos agravos decorrentes desse tipo de violência, implica o desenvolvimento de parcerias efetivas, principalmente com a justiça e a segurança pública.

A prevenção nas escolas é essencial para disponibilizar informações sobre DST/aids, sexualidade, gravidez e violência aos jovens em geral, não devendo nos esquecer daqueles que não estão vinculados às instituições de ensino.

O combate ao racismo institucional é outra seríssima questão a ser enfrentada pelo Estado. Começando pelo seu reconhecimento e perpassando por discussões e capacitações para os profissionais das instituições, que sensibilizados e alertados, poderiam modificar suas atuações, levando a uma diferente forma de relação entre os equipamentos sociais e a população negra.

0 racismo institucional precisa ser explicitado e combatido por meio de várias ações afirmativas em relação à mulher negra, visando à promoção da equidade em saúde (Cruz, 2004). São necessárias intervenções mais incisivas, não apenas no campo da informação e prevenção das DST/aids, mas incorporar parcerias com diversos setores e instituições, pois apenas uma ação multifacetada pode caminhar no sentido de modificar a realidade hoje imposta.

Saliente-se que a parceria com a sociedade civil é fundamental para fortalecer os mecanismos de controle social, sem o qual as propostas de mudança de paradigmas se perdem no tempo e no espaço.

Uma das principais abordagens de êxito utilizadas na educação para a prevenção entre mulheres nasceu do movimento pelos direitos da mulher, que trouxe o conceito de "empowerment", que pode ser traduzido como "fortalecimento para a mudança" ou "empoderamento", através do qual se discute que a mudança de comportamento, para um comportamento mais seguro, não é resultado apenas de conscientização dos riscos a que as pessoas estão submetidas, mas passa por pressões sociais e por recursos econômicos, culturais, jurídicos e outros, que são historicamente distribuídos de maneira desigual entre gêneros, gerações e segmentos populacionais, quer por sua inserção social, quer por seu pertencimento étnico-racial.

No entanto, apesar do panorama sombrio, alguns países africanos têm conseguido diminuir as taxas de infecção pelo HIV claramente vinculadas a programas de prevenção sólidos, com a promoção do uso de preservativo, regulamentação do sexo comercial, tratamento de doenças sexualmente transmissíveis e mobilização da comunidade. Uganda conseguiu reduzir a prevalência de HIV de quase $13 \%$ em 1994 a 9,5\% em 1997. Em Masaka, Uganda, a prevalência de HIV em mulheres de 20 a 24 anos decresceu de 20,9\% em 1989/1990 a 13,8\% em 1996/1997. Na Zâmbia, em mulheres assistidas em clínicas de pré-natal, de 15 a 19 anos, a soroprevalência passou de 27\% em 1993 a 17\% em 1998. Mesmo no mundo industrializado, não se tem uma redução dessa magnitude, calcada em programas de prevenção (Santos, 2002).

Os avanços da terapia antirretroviral e as tendências atuais da epidemia, concentrando-se em países e populações mais socialmente vulneráveis, devem levar a novas reflexões sobre as formas de abordagem do HIV/aids sob suas diversas nuanças de gênero e étnico-raciais.

É importante incentivar a testagem anti-HIV da população negra como um todo e das mulheres negras em particular, sem, entretanto, incorrer no erro de estigmatizá-las ainda mais (Lopez, 2011). A realização ampliada de testes favorece o diagnóstico precoce da infecção pelo HIV, o que pode impactar sobremaneira o prognóstico dessa infecção.

Deve-se ampliar os projetos de prevenção para os segmentos da população especialmente vulneráveis, como é o caso das mulheres negras, desde as ações 
de promoção da saúde e prevenção primária até o investimento no trabalho de adesão ao tratamento com antirretrovirais para as pessoas já diagnosticadas. Esse seria um caminho para diminuir a mortalidade específica por aids.

É fundamental o cuidado integral às pessoas negras vivendo com HIV/aids, com a implementação e manutenção de políticas públicas de prevenção e assistência cada vez mais adequadas e eficientes, com o desafio e a obrigação moral e ética de tornálas acessíveis a toda a população (Greco, 1999; Kalckmann, 2007).

O poeta negro Aimé Cesaire disse que "as duas maneiras de perder-se são: por segregação, sendo enquadrado na particularidade, ou por diluição no universal". A utopia que hoje perseguimos consiste em buscar um atalho entre uma negritude redutora da dimensão humana e a universalidade ocidental hegemônica que anula a diversidade. Ser negro sem ser somente negro, ser mulher sem ser somente mulher, ser mulher negra sem ser somente mulher negra. Alcançar a igualdade de direitos é converter-se em um ser humano pleno e cheio de possibilidades e oportunidades para além de sua condição de raça e de gênero. Esse é o sentido final dessa luta. (Carneiro, 2011, p. 6)

A luta feminista e antirracista, efetuada pelas mulheres negras de todo o mundo, está calcada na crença de que é possível construir uma sociedade mais fraterna e solidária, onde as diversidades étnico-raciais e culturais sejam respeitadas, e os "diferentes sejam entendidos como iguais" (Carneiro, 2003).

A introdução das questões relativas a gênero e pertencimento étnico-racial na agenda das políticas públicas em geral levará a sociedade a um fortalecimento da democracia, à igualdade e justiça social (Carneiro, 2003).

No campo particular da saúde, apenas com uma visão ampliada sobre os determinantes do adoecimento e morte dos diversos segmentos populacionais, com uma gama de ações multissetoriais, com incisivo enfrentamento do racismo institucional pelo Estado e com o fortalecimento do movimento social, será possível iniciar a jornada para se alcançar a longínqua equidade na saúde

Urge um novo olhar!

\section{Referências}

ADORNO, R. C. F.; ALVARENGA, A. T.;

VASCONCELLOS, M. P. Quesito cor no sistema de informação em saúde. Estudos Avançados, São

Paulo, v. 18, n. 5o, p. 119-123, 2004.

ALBUQUERQUE, V. S.; MOÇO, E. T. S. M.;

BATISTA, C. M. Mulheres negras e HIV:

determinantes de vulnerabilidade na região serrana do estado do Rio de Janeiro. In: CAMPOS, A. C. M.; ALMEIDA, C. R.; AOKI, F. H. (Org.). Saúde da população negra, HIV/AIDS: pesquisas e práticas. Campinas: Arte escrita, 2011. p. 101-122.

AYRES, J. C. R. M. et al. O conceito de vulnerabilidade e as práticas de saúde: novas perspectivas e desafios. In: CZERESNIA, D.; FREITAS, C. M. Promoção da saúde: conceitos, reflexões, tendências. Rio de Janeiro: Fiocruz, 2003. p. 117-139.

BARBOSA, R. H. S. AIDS e saúde reprodutiva: novos desafios. In: GIFFIN, K.; COSTA, S. H. (Org.). Questões de saúde reprodutiva. Rio de Janeiro: Fiocruz, 1999. p. 281-296.

BASTOS, F. I.; SZWARCWALD, C. L. AIDS e pauperização: principais conceitos e evidências empíricas. Cadernos de Saúde Pública, Rio de Janeiro, v. 16, supl. 1, p. 65-76, 2000.

BASTOS, F. I. Taxas de infecção de HIVe sífilis e inventário de conhecimento, atitudes e práticas relacionadas às infecções sexualmente transmissiveis entre usuários de drogas em 10 municípios brasileiros. Relatório técnico entregue ao Departamento de DST, AIDS e Hepatites Virais, 2009.

BATISTA, L. E. Mulheres e homens negros: saúde, doença e morte. 2002. Tese (Doutorado em Sociologia) - Faculdade de Ciências e Letras da UNESP, Araraquara, 2002.

BLAKE, M. T. et al. Characteristics of sexual violence against adolescent girls and adult 
women. BMC Women's Health, London, v. 14, n. 15, 2014 .

BRASIL. Ministério da Saúde. Portaria nº 992, de 13 de maio de 2009. Política nacional de atenção integral a saúde da população negra. Diário Oficial da União, Brasília, DF.

BRASIL. Ministério da Saúde. Secretaria de Vigilância Sanitária. Programa Nacional de Controle e Prevenção de DST/AIDS. Boletim epidemiológico: Aids DST. 2011. Disponível em: <http://www.aids. gov.br/publicacao/2011/boletim epidemiologico_2011>. Acesso em: 12 nov. 2013.

CAMPBELL, J. C. et al. The intersection of intimate partner violence against women and HIV/AIDS: a review. International Journal of Injury Control and Safety Promotion, Baltimore, v. 15, n. 4, p. 221-231, 2008.

CARNEIRO, S. Mulheres em movimento. Estudos Avançados, São Paulo, v. 17, n. 49, p. 117-132, 2003.

CARNEIRO, S. Enegrecer o feminismo: a situação da mulher negra na América Latina a partir de uma perspectiva de gênero. 2011. Disponível em: <http://arquivo.geledes.org.br/em-debate/ sueli-carneiro/17473-sueli-carneiro-enegrecero-feminismo-a-situacao-da-mulher-negra-naamerica-latina-a-partir-de-uma-perspectiva-degenero>. Acesso em: 29 mar. 2016.

CRENSHAW, K. Documento para o encontro de especialistas em aspectos da discriminação racial relativos ao gênero. Estudos Feministas, Los Angeles, ano 10, n. 1, p. 171-188, 2002.

CRUZ, I. C. F. A sexualidade, a saúde reprodutiva e a violência contra a mulher negra: aspectos de interesse para assistência de enfermagem. Revista da Escola de Enfermagem da USP, São Paulo, v. 38, n. 4, p. 448-457, 2004.

CUNHA, E. M. G. P. Diferenças étnico-raciais nas características sociodemográficas da população paulista. In: KALCKMANN, S. et al. (Org.). Nascer com equidade, humanização do parto e do nascimento: questões raciais/cor e gênero. São Paulo: Instituto de Saúde, 2010, p. 85-110.
DIAS, J.; GIOVANETTI, M. R.; SANTOS, N. J. S. Qual é a sua cor ou raça/etnia?: perguntar não ofende - responder ajuda a prevenir. São Paulo: SES - CCD, Centro de Referência e Treinamento DST/AIDS, 2009.

DINIZ, N. M. F. et al. Mujeres víctimas de la violencia sexual: adhesión a la quimioprofilaxia del HIV. Revista Latino-Americana de Enfermagem, Ribeirão Preto, v. 15, n. 1, 2007.

DUNKLE, K. L. et al. Gender-based violence, relationship power, and risk of HIV infection in women attending antenatal clinics in South Africa. London, v. 363, n. 9419, p. 1415-1421, 2004.

GARCIA, S.; SOUZA, F. M. Vulnerabilidades ao HIV/AIDS no contexto brasileiro: iniquidades de gênero, raça e geração. Saúde e Sociedade, São Paulo, v. 19, supl. 2, p. 9-20, 2010.

GARCIA-MORENO, C. et al. Prevalence of intimate partner violence: findings from the WHO multicountry study on women's health and domestic violence. Lancet, v. 368, n. 9543, p. 1260-1269, 2006.

GIFFIN, K. Beyond empowerment: heterosexualities and the prevention of AIDS. Social Science \& Medicine, v. 46, n. 2, p. 151-156, 1998.

GRECO, D. B. Ética, saúde e pobreza: as doenças emergentes no século XXI. Bioética, Brasília, DF, v. 7, n. 2, p. 189-198, 1999.

GUIBU, I. A.; TAYRA, Â.; CARMO, M. C. P. Panorama da AIDS no estado de São Paulo. In: WALDVOGEL, B. G.; TAYRA, Â.; GUIBU, I. A. Dados para repensar a AIDS no Estado de São Paulo: resultados da parceria entre Programa Estadual de DST/AIDS e Fundação Seade. São Paulo: Fundação Seade, 2010. p. 25-58.

KALCKMANN, S. et al. Racismo institucional: um desafio para a equidade no SUS? Saúde e Sociedade, São Paulo, v. 16, n. 2, p. 146-155, 2007.

KERR, L. Comportamento, atitudes, práticas e prevalência de HIV e sífilis entre homens que fazem sexo com homens (HSH) em 10 cidades brasileiras. Relatório técnico entregue ao Departamento de DST, AIDS e Hepatites Virais, 2009. 
KRONBAUER, J. F. D.; MENEGHEL, S. N. Perfil da violência de gênero perpetrada por Companheiro. Revista de Saúde Pública, São Paulo, v. 39, n. 5, p. 695-701, 2005.

LOPES, F. et al. Prevalência de HIV, papilomavírus humano e sífilis na Penitenciária Feminina da Capital, São Paulo, 1997-1998. Cadernos de Saúde Pública, Rio de Janeiro, v. 17, n. 6, p. 1473-1480, 2001.

LOPES, F. Mulheres negras e não negras vivendo com HIV/AIDS no Estado de São Paulo: um estudo sobre suas vulnerabilidades. 2003. Tese (Doutorado em Saúde Pública), Faculdade de Saúde Pública da Universidade de São Paulo, São Paulo, 2003.

LOPEZ, L. C. Uma análise das políticas de enfrentamento ao HIV/AIDS na perspectiva da interseccionalidade de raça e gênero. Saúde e Sociedade, São Paulo, v. 20, n. 3, p. 590-603, 2011.

MANN, J. M.; TARANTOLA, D. J. M.; NETTER, T. W. A AIDS no mundo. Rio de Janeiro: RelumeDumará, 1993.

MARTIN, S. L.; CURTIS, S. Gender-based violence and HIV/AIDS: recognising links and acting on evidence. Lancet, v. 363, n. 9419, p. 1410-1411, 2004.

MENEGHEL, S. N. ; FARINA, O. ; RAMÃO, S. R. Histórias de resistência de mulheres negras. Estudos Feministas, Florianópolis, v. 13, n. 3, p. 567-583, 2005 .

OLIVEIRA, M. L. P.; MENEGHEL, S. N.; BERNARDES, J. S. Modos de subjetivação de mulheres negras: efeitos da discriminação racial. Psicologia \& Sociedade, v. 21, n. 2, p. 266-274, 2009.

PHORANO, O.; NTHOMANG, K.; NTSEANE, D. Alcohol abuse, gender-based violence and HIV/ AIDS in Botswana: establishing the link based on empirical evidence. Journal of Social Aspects of HIV/AIDS: An Open Access Journal, v. 2, n. 1, p. 188-202, 2012.

PINHO, M. D. et al. Juventudes, raça e vulnerabilidades. Revista Brasileira de Estudos de População, v. 19, n. 2, p. 277-294, 2002.

RISCADO, J. L. S.; OLIVEIRA, M. A. B.; BRITO, A. M. B. Vivenciando o racismo e a violência: um estudo sobre as vulnerabilidades da mulher negra e a busca de prevenção do HIV/AIDS em comunidades remanescentes de quilombos, em Alagoas. Saúde e Sociedade, São Paulo, p. 96-108, 2010.

RONALD, A. R. Slowing heterosexual HIV transmission. Infectious Disease Clinics of North America, v. 9, n. 2, p. 287-296, 1995.

SANTOS, N. J. S. As Mulheres e suas decisões reprodutivas, diante da Epidemia de HIV/AIDS. 2002. Tese (Doutorado em Saúde Pública), Faculdade de Saúde Pública da Universidade de São Paulo, São Paulo, 2002.

SANTOS, N. J. S. et al. Contextos de vulnerabilidade para o HIV entre mulheres brasileiras. Cadernos de Saúde Pública, Rio de Janeiro, v. 25, p. 321-333, 2009. Suplemento 2.

SANTOS, S. M.; GUIMARÃES, M. J. B.; ARAÚJO, T. V. B. Desigualdades raciais na mortalidade de mulheres adultas no Recife, 2001 a 2003. Saúde e Sociedade, São Paulo, v. 6, n. 2, p. 87-102, 2007.

SÃO PAULO (Estado). Secretaria de Estado da Saúde. Boletim Epidemiológico: C. R. T. - DST/ AIDS. C. V. E. Ano XXXI, n. 1, 2014.

SÃO PAULO (Município). Programa Municipal de DST/ AIDS. Boletim Epidemiológico de AIDS, HIV e DST, ano XVI, n 16, junho, 2012.

SCHRAIBER, L. B. et al. Violência contra a mulher: estudo em uma unidade de atenção primária à saúde. Revista de Saúde Pública, São paulo, 36, n. 4, p. 470477, 2002.

SCHRAIBER, L. B.; D’OLIVEIRA, A. F. P. L.; FRANÇA JUNIOR, I. Violência sexual por parceiro íntimo entre homens e mulheres no Brasil urbano, 2005. Revista de Saúde Pública, São Paulo, v. 42, pg. 127-137, 2008. Suplemento 1.

SILVERMAN, J. G. et al. Violence against wives, sexual risk and sexually transmitted infection among Bangladeshi men. Sexually Transmitted Infections Journal, v. 83, n. 3, p. 211-215, 2007.

SZWARCWALD, C. L. et al. HIV testing during pregnancy: use of secondary data to estimate 2006 test coverage and prevalence in Brazil. 
Brazilian Journal of Infectious Diseases, v. 2, n. 3 , p. 167-172, 2008.

SZWARCWALD, C. L. Taxas de prevalência de HIV e sifilis e CAP de risco relacionadas às IST nos grupos de mulheres profissionais do sexo, no Brasil. Relatório técnico entregue ao Departamento de DST, Aids e Hepatites Virais, 2009.

TAQUETTE, S. R. Vulnerabilidade às DST/AIDS de adolescentes femininas afrodescendentes moradoras de comunidades pobres do município do Rio de Janeiro. In: CAMPOS, A. C. M.; ALMEIDA, C. R. A.; AOKI, F. H. (Org.). Saúde da população negra, HIV/AIDS: pesquisas e práticas. Campinas: Arte Escrita, 2011. p. 43-54.
TAQUETTE, S. R.; TEIXEIRA, S. A. M. AIDS e juventude: gênero, classe, raça. Rio de Janeiro; UERJ, 2009.

TAQUETTE, S. R.; TEIXEIRA, S. A. M. Violência e atividade sexual desprotegida em adolescentes menores de 15 anos. Revista da Associação Médica Brasileira, São Paulo, v. 56, n. 4, p. 440446, 2010.

VERMELHO, L. Women with AIDS: transmission and prevention strategies. In: 12TH WORLD AIDS CONFERENCE, 28 jun./3 jul. Anais... Geneva, 1999. VOLOCHKO, A. Mortalidade materna: determinantes sociopolíticos. In: KALCKMANN, S. et al. (Org.). Nascer com equidade, humanização do parto e do nascimento: questões raciais/cor e gênero. São Paulo: Instituto de Saúde, 2010, p. 111-132.

\section{Agradecimentos}

Agradecimentos ao Sr. Sergio Giannella, da Faculdade de Saúde Pública da Universidade de São Paulo, pela coleta e organização dos dados do SIA-SUS (2012).

Recebido: $11 / 04 / 2014$ Aprovado: 17/04/2014 